\title{
Fundamentals of Knowledge Technology
}

\author{
Preface
}

This issue of the Fundamenta Informaticae presents some selected and extended papers originally scheduled for the Rough Sets and Knowledge Technology conference in Chengdu, China, May 17 19, 2008. These papers, related to Fundamentals of Knowledge Technology, cover an entire spectrum of theoretical and application-oriented trends of rough set theory. Specifically, starting from theoretical approaches, two papers (Approximation Algebra and Framework by D. Ciucci and Pairwise Comparisons Based Non-Numerical Ranking by R. Janicki) describe generalized approximation algebras and deriving weakly ordered non-numerical rankings, respectively. The next two papers (Semi-supervised Rough Cost/Benefit Decisions by P. Lingras et al. and An Incremental Approach for Inducing Knowledge from Dynamic Information Systems by D. Liu et al.) discuss financial cost/benefit decisions and so called interesting knowledge. Another pair of papers (Dominance-Based Rough Sets Using Indexed Blocks as Granules by C. Chan and G. Tzeng and Learning Rule Ensembles for Ordinal Classification with Monotonicity Constraints by K. Dembczynski et al.) cover dominance relations. Two papers (A TwoPhase Model for Learning Rules from Incomplete Data by $\mathrm{H}$. Li et al. and An Integration of Cloud Transform and Rough Set Theory to Induction of Decision Trees by J. Song et al.) show how to mine incomplete data. The paper An Extended Comparison of Six Approaches to Discretization-A Rough Set Approach by P. Blajdo et al.) compares six approaches to discretization. Finally, the paper Single $2 D$ Image-based 3D Face Reconstruction and Its Application in Pose Estimation by X. Gong et al. is related to face recognition.

We would like to thank Dr. A. Skowron for his guidance during the process of editing this issue.

\section{Guest editors}

Jerzy W. Grzymala-Busse (University of Kansas, Polish Academy of Sci.) Guoin Wang (Chongqing University of Posts and Telecommunications) Yiyu Yao (University of Regina) Wojciech Ziarko (University of Regina) 